\title{
CRISTINA REDONDO SOBRE RAZONES Y NORMAS
}

José Juan Moreso

Universidad Pompeu Fabra, Barcelona

(I)

En el trabajo de M.C. Redondo 'Razones y normas' ${ }^{1}$ se hallan presentadas, de un modo especialmente perspicuo, dos concepciones incompatibles de la racionalidad práctica: el universalismo y el particularismo. Delimitadas ambas posiciones se ofrece una muy atinada reconstrucción del problema y de su repercusión en el ámbito de las normas jurídicas concebidas como razones para la acción.

El trabajo está repleto de consideraciones valiosas y de cuestiones incidentales tratadas con agudeza suma. Sin embargo, voy a limitar este comentario a tres cuestiones planteadas por la autora: el trasfondo metafísico de la polémica entre universalismo y particularismo, las tres nociones de universalidad de las normas presentadas en el trabajo y, por último, un dilema que la autora plantea a Dworkin y al positivismo jurídico inclusivo (p. 57) y, que dicho ahora de modo muy simplificado, plantea la necesidad de que estas concepciones renuncien a la universalidad o bien de las razones jurídicas o bien de las razones morales. Al hilo de este dilema tendré ocasión de plantear aquella concepción más adecuada, en mi opinión, de las razones morales y de las razones jurídicas y, también, su lugar en el razonamiento práctico.

${ }^{1}$ María Cristina Redondo, 'Razones y normas', en este número. Una versión ligeramente diversa: 'Legal Reasons' en IVR 21st World Congress, 12-18 August 2003, Lund, Sweden. 
Redondo (p. 28) afirma:

El actual debate entre universalismo y particularismo en el ámbito de la filosofía práctica es, en gran medida, una discusión metafísica sobre la posibilidad de establecer relaciones nomológicas entre ciertas propiedades naturales, por una parte, y ciertas propiedades morales por otra o, también, acerca de la fuente de la relevancia moral de ciertas propiedades naturales.

No es que esté en desacuerdo con esta afirmación, pero creo que debe entenderse adecuadamente, si se me permite decirlo así, debe ser entendida con poca carga metafisica. Principalmente para hacerla compatible con otra afirmación (p. 31) de la autora conforme a la cual 'una concepción universalista de las normas es compatible con el realismo, el anti-realismo, el cognoscitivismo, el no-cognoscitivismo, etc.'.2 Para hacer ambas afirmaciones compatibles es preciso entender de un modo adecuado la primera de ellas. En sentido estricto, los antirrealistas y los no-cognoscitivistas en moral no creen que haya nada como propiedades morales, los predicados morales se refieren si acaso a proyecciones de los seres humanos sobre el mundo a partir de sus evaluaciones. ${ }^{3}$ Es decir, la presunta superveniencia (a la que la autora se refiere en p. 48) será entendida de modos muy diversos, según la concepción metaética que los autores asuman. Es por esta razón que creo que el debate es mejor configurado como un debate entre dos concepciones diversas de la racionalidad práctica que como una disputa metafísica. Para los realistas morales dicha disputa es sólo un reflejo de una

${ }^{2}$ Vd., también citado por la autora, Russ Shafer-Landau, 'Moral Rules', Ethics 107 (1997): 584-611, en pp. 584-585.

${ }^{3}$ Vd., por ejemplo, Simon Blackburn, 'Morals and Modals' en Simon Blackburn, Essays in Quasi-Realism, (Oxford: Oxford University Press, 1993), 52-74. 
disputa ontológica acerca de cómo están constituidas las propiedades del mundo; para los antirrealistas, en cambio, la disputa es acerca de cuál es la reconstrucción más adecuada del discurso práctico, sin implicaciones acerca de lo que hay en el mundo, acerca de lo que hay out there. No es que haya, tal como lo comprendo, una discrepancia en este punto entre lo que pienso y las ideas de la autora, pero considero conveniente quitar hierro a la dimensión metafísica de esta polémica.

\section{(III)}

Redondo (p. 33) distingue tres sentidos en los que podemos decir que una norma es universal: generalidad semántica, universalidad lógica y relevancia universal.

Comencemos por los dos primeros sentidos de universalidad y, sólo después de analizados, pasaremos al tercero. Por generalidad semántica, la autora entiende que una norma es en este sentido universal cuando se refiere a una clase de circunstancias y no a un caso particular. Desde este punto de vista, la generalidad semántica es una propiedad gradual. En cambio, la universalidad lógica para la autora depende de que la forma lógica de las normas sea la de un condicional universalmente cuantificado, y no la de un condicional derrotable (defeasible).

Sin embargo, los enunciados de razón y las normas son siempre, en el primer sentido, universales. Nunca se refieren a un caso particular, a un evento describible mediante una constante, para decirlo con los términos de la lógica. Por ejemplo, el enunciado: 'José Juan Moreso debe llevar un regalo a su hija de su viaje a Génova' es más particular que el enunciado 'Los padres deben llevar regalos a sus hijos cuando viajan'. Sin embargo, lo que es obligatorio son acciones genéricas, no acciones individuales. Toda obligación, moral o de otro tipo, puede ser cumplida por más de un acto individual. No hace falta acudir al debate sobre el particularismo para 
establecer esta cuestión, basta con el análisis de los enunciados de acciones, que han de ser analizados siempre mediante cuantificadores. ${ }^{4}$ Por lo tanto, si el particularismo es entendido como la concepción según la cual todos los enunciados morales son básicamente enunciados acerca de acciones particulares, entonces este tipo de particularismo es conceptualmente imposible. ${ }^{5}$

Como R.M. Hare nos recuerda, ${ }^{6}$ mientras el término 'general' se opone a 'específico', el término 'universal' se opone a 'singular'. Los enunciados de razón pueden ser más o menos generales, pero siempre son, en relación con las acciones, universales. También Carlos E. Alchourrón nos lo recuerda: ${ }^{7}$

Sin embargo, los enunciados de razón, cualquiera sea el tipo de razón considerada (suficiente, necesaria, contribuyente, sustitutiva, etc.), no sólo son enunciados condicionales sino que además son enunciados generalizados universalmente. Este aspecto ha pasado con frecuencia inadvertido porque, en los ejemplos con que se ilustra la teoría de las razones, figuran referencias a individuos particulares (yo y mi hijo) y a momentos determinados (el día del cumpleaños de mi hijo) sin que se requiera referencia a todos los individuos o todos los momentos de cierta totalidad. Esto es verdad: un enunciado de razón no tiene por qué ser general respecto de los individuos y los

${ }^{4}$ Como muestra el conocido análisis de Donald Davidson, 'The Logical Form of Action Sentences' en Donald Davidson, Essays on Actions and Events (Oxford: Oxford University Press, 1980), 105-148.

${ }^{5}$ Con estos argumentos y con el nombre de 'particularismo analítico' ha sido recientemente criticado por Walter Sinnot-Armstrong, 'Some Varieties of Particularism', Metaphilosophy, 30 (1999): 1-12, en p. 2.

${ }^{6}$ R.M. Hare, Freedom and Reason (Oxford: Oxford University Press, 1963), pp. 38-40.

${ }^{7}$ Carlos E. Alchourrón, 'Para una lógica de las razones prima facie', Análisis Filosófico, 16 (1996): 113-124, en p. 118. 
momentos considerados. Ninguna de estas formas de generalidad es requerida en cada enunciado de razón. Sin embargo, hay un tipo de generalidad que no puede faltar, esto es: la generalidad universal respecto de las circunstancias posibles consideradas.

Esta cita de Alchourrón es relevante porque afecta al segundo sentido, el sentido lógico de universalidad, propuesto por Redondo, que apoyándose en Alchourrón dice (p. 33):

una norma puede ser universal en un sentido lógico... cuando la forma lógica atribuida a una norma es la de un condicional universalmente cuantificado, como opuesta a la de un condicional derrotable (defeasible).

La cuantificación universal de las circunstancias a la que Alchourrón se refiere es un rasgo de todos los enunciados de razón. Esto conlleva que los enunciados de razón deben ser representados mediante el uso del condicional estricto de la lógica modal, que es la modalización con el operador de necesidad de un condicional material y el operador de necesidad no es otra cosa que la cuantificación universal respecto de las circunstancias. No representa, en Alchourrón, ninguna diferencia relevante entre condicionales derrotables y condicionales inderrotables, puesto que para Alchourrón, los condicionales derrotables (los enunciados de razón prima facie) son analizables mediante condicionales inderrotables. Los condicionales derrotables son representados así por Alchourrón:

$$
\mathrm{A}>\mathrm{B} \text {, }
$$

en donde $\mathrm{A}$ es una condición, prima facie, de B. Esto no significa que siempre que se da $\mathrm{A}$ se da $\mathrm{B}$, porque $\mathrm{A}$ puede estar acompañado de otras circunstancias que hacen que A plus $\mathrm{C}$, por ejemplo, no produzcan B. Por ejemplo, aunque podemos aceptar una generalización como 'los pájaros vuelan', los pájaros que son pingüinos no vuelan; y aunque el hecho de 
haber prometido cenar con un amigo mañana es una razón para que yo acuda mañana a un determinado restaurante, si mi hija es ingresada en el hospital entonces yo tal vez no deba acudir a dicho restaurante mañana. Por esta razón, Alchourrón afirma que para los condicionales derrotables no son válidas ni las reglas lógicas del refuerzo del antecedente (cuando 'si A entonces es B' es válida, entonces también lo es 'Si A y C, entonces B') ni el modus ponens (la validez de 'A entonces B' y 'A' implican 'B'). ${ }^{8}$ Ahora bien, la definición de Alchourrón de los condicionales derrotables es la siguiente: ${ }^{9}$

$$
(\text { Def. }>)(\mathrm{A}>\mathrm{B})=(f \mathrm{~A} \Rightarrow \mathrm{B})
$$

La expresión ' $(f \mathrm{~A} \Rightarrow \mathrm{B})$ ', que contiene el condicional estricto, es equivalente a (siendo ‘ ’ el operador moral de necesidad)

- $(f \mathrm{~A} \rightarrow \mathrm{B})$,

es decir la necesitación de un condicional material. El operador ' $f$ ' es un operador de revisión, que transforma el antecedente de un condicional derrotable en un antecedente de un condicional estricto, para el que valen las reglas del refuerzo del antecedente y del modus ponens. Por lo tanto, para Alchourrón el condicional derrotable es sólo un condicional inderrotable enmascarado, que en su definición contiene un condicional universalizado acerca de las circunstancias. En esta reconstrucción no hay espacio para distinguir entre universalismo y particularismo. Un modo, sin embargo, en que el particularismo moral puede ser presentado con los términos de Alchourrón es el siguiente: los enunciados de razones morales no admiten, por razones conceptuales, una revisión consistente, es decir, no hay modo de establecer las propiedades que deben acompañar a un antecedente de un principio moral para determinar que, en su presencia, es válida la obligación del consecuente. No hay modo de establecer puentes entre las propiedades naturales mencionadas en el antecedente y las propiedades morales del

\footnotetext{
${ }^{8}$ Ibidem, pp.115-116.

${ }^{9}$ Ibidem, pp. 122.
} 
consecuente. Esto sería otro modo de sostener que las propiedades relevantes que determinan la corrección e incorrección moral son 'naturally shapeless'. ${ }^{10}$ Volveré sobre ello más adelante.

Vayamos ahora al tercer sentido de universalidad de Redondo. Según Redondo, este tercer sentido (p. 34)

hace referencia a la relevancia de las normas en nuestra deliberación práctica, moral o jurídica... Este sentido de universalidad, no es parte necesaria del concepto universalista de norma, y presupone que la norma es válida o está justificada.

Para la concepción universalista, determinadas propiedades son uniforme e invariablemente relevantes. Para la concepción particularista, 'ninguna propiedad es uniforme e invariablemente relevante' (p.35). Estoy de acuerdo con esta última afirmación, pero no sé qué relación guarda esta última afirmación con la referida a la relevancia de las normas en nuestra deliberación práctica. De un modo similar al que la lógica proposicional sólo garantiza que una conclusión de una inferencia válida es verdadera si las premisas de la inferencia también lo son, que una conclusión deóntica es válida sólo está garantizado si las premisas (las normas) de la inferencia son válidas. Las premisas falsas no garantizan la verdad de las conclusiones en inferencias válidas, del mismo modo las normas inválidas no garantizan la validez de las conclusiones en inferencias válidas. No creo que nadie, universalista ni particularista, se oponga a esta afirmación. Lo que el particularista desafía es que pueda haber normas válidas, precisamente.

Es cierto que Redondo (p. 37) propone dos modos de reconstruir el particularismo: a) como que no existen normas universales justificadas, es decir que las normas universales carecen de relevancia práctica y b) como

\footnotetext{
${ }^{10}$ Como sostienen los particularistas, vd. Jonathan Dancy, Moral Reasons, (Oxford: Blackwell, 1993), p. 76. Otras interesantes presentaciones del particularismo pueden hallarse en David McNaughton, Moral Vision, (Oxford: Oxford Blackwell, 1988) y John McDowell, Mind, Value, and Reality (Cambridge; Harvard University Press, 1998).
} 
una concepción de las normas como condicionales derrotables. Redondo descarta la primera posibilidad aduciendo que no parece razonable concebir a los particularistas como 'universalistas escépticos' y por ello se decanta por la segunda posibilidad. Sin embargo, las anteriores reflexiones, fundadas en los trabajos de Alchourrón, ${ }^{11}$ muestran que no basta para el particularismo concebir las normas como el contenido de condicionales derrotables, sino que debe añadirse que dichos condicionales no puedan, por razones conceptuales, ser sujetos a revisión. En este sentido, las dos propuestas de reconstrucción de la autora son equivalentes, puesto que si las normas son condicionales derrotables no revisables, entonces no hay normas válidas universalmente justificadas, es decir no hay normas cuyo contenido son condicionales inderrotables.

En síntesis, creo que ninguno de los tres sentidos de universalidad y sus negaciones propuestos por la autora son capaces de capturar el núcleo del debate entre universalismo y particularismo, puesto que el sentido semántico de universalidad es trivial: no hay enunciados de razón, por razones conceptuales, acerca de acciones individuales; el sentido lógico tampoco discrimina entre razones, puesto que todas las razones del tipo que fueren comportan una cuantificación universal de las circunstancias y la universalidad de la relevancia sólo nos dice que únicamente pueden constituir razones las normas válidas. Creo que un particularista podría aceptar las tres cosas. Lo que el particularista desafía, como la autora pone de manifiesto, es la invariabilidad y uniformidad de las propiedades relevantes. Como he sostenido, en los términos de Alchourrón, este punto puede ser capturado diciendo no

${ }^{11}$ Vd. también, aparte del trabajo citado en nota 7, Carlos E. Alchourrón, 'Philosophical Foundations of Deontic Logic and the Logic of Defeasible Conditionals' en J.J. Meyer, R.J. Wieringa (eds.), Deontic Logic in Computer Sciences: Normative System Specifications (New York: Wiley \& Sons, 1993), 43-84; 'Defeasible Logics: Demarcation and Affinities' en G. Grocco, L. Fariñas del Cerro, A. Herzig (eds.), Conditionals: from Philosophy to Computer Science (Oxford: Oxford University Press, 1995), 67-102; 'Detachment and Defeasibility in Deontic Logic', Studia Logica, 57 (1996): 5-18 
sólo que las normas son como máximo condicionales derrotables, sino añadiendo que en el ámbito moral no pueden ser sometidos, por razones conceptuales, a revisión. Por lo tanto, universalistas y particularistas pueden aceptar que hay normas o principios morales concebidos como condicionales derrotables, en lo que difieren es en lo siguiente: los universalistas afirman que dichos condicionales pueden ser sometidos a revisión, de modo consistente, y dan lugar a normas o principios morales inderrotables; los particularistas niegan esta afirmación.

Antes de ocuparme del dilema que la autora plantea a aquellas concepciones jurídicas que piensan que la moral puede representar un papel destacado en la identificación de aquello que el derecho requiere y en el razonamiento jurídico, considero conveniente establecer mi propia posición personal acerca del debate entre el universalismo y el particularismo en los términos planteados, es decir, acerca de la posibilidad de revisar consistentemente los antecedentes de nuestras normas o principios morales.

La estrategia particularista es siempre del siguiente tenor: un universalista propone una norma moral como: 'se deben cumplir las promesas', entonces el particularista trata de mostrarle que el hecho de haber prometido hacer A no siempre constituye una razón para hacer A. El particularista puede replicar, ¿es una razón para matar a una persona el hecho de haberlo prometido a cambio de un millón de euros? Ante esta réplica, el universalista puede contrarreplicar de dos modos: uno muy implausible: aceptar que constituye una razón, otro más razonable: reformular la norma moral propuesta para que no incluya estos supuestos, por ejemplo: 'Se deben cumplir las promesas que no conllevan la realización de comportamientos inmorales'. Ahora el particularista vuelve a la carga: ¿qué ocurre si la promesa ha sido obtenida bajo la amenaza de secuestrar a la hija del que ha prometido? Y el universalista replica con una nueva formulación: 'se deben cumplir las promesas que no 
han sido otorgadas bajo amenaza ni conllevan la realización de actos inmorales.' El particularista todavía puede desafiar diciendo que si arrancamos de un torturador, bajo amenaza, la promesa de dejar de torturar, entonces debe dejar de torturar y el universalista puede añadir el adjetivo 'injustas' al sustantivo 'amenazas'. Y así sucesivamente.

Es decir, ante el embate particularista, el universalista sólo tiene una estrategia razonable: la estrategia de la especificación de los principios y normas morales. ${ }^{12}$

Considero la estrategia especificacionista razonable. Sin embargo, para que dicha estrategia sea convincente debe ser sometida a dos limitaciones importantes. Por una parte, como el ejemplo anterior sugiere, dicha especificación contendrá siempre, entre las circunstancias que permiten revocar la obligación moral a la que en principio llevan determinadas propiedades -llamémosle defeaters- ${ }^{13}$ conceptos morales. Supongamos que yo he prometido ir esta noche a cenar a casa de un amigo. Si mi amigo me llama para decirme que si no termino el artículo que estoy escribiendo puedo no ir a su casa, este hecho socava la razón para cumplir la promesa, se trata de un underminer. Ahora bien, si mi amigo me llama para decirme que está muy cansado y que no se siente con fuerzas para cocinar esta noche, entonces este hecho cancela mi obligación de un modo diverso al anterior: no sólo la socava sino que me ofrece una razón para no ir a su casa, se trata de un reverser. Y si yo sufro mareos y no me encuentro bien para ir a su casa, entonces mi responsabilidad es anulada mediante una excusa. Por otra parte, si es mi mujer la que me llama desde el hospital en el que ha sido ingresada

\footnotetext{
${ }^{12}$ Una estrategia sugerida por R.M. Hare, The Language of Morals (Oxford: Oxford University Press, 1953), pp. 48-55 y pp. 60-65. Vd., también, por ejemplo, Russ Shafer-Landau, 'Specifying Absolute Rights', Arizona Law Review 37 (1995). 209-225 y, en el contexto de discusión del particularismo, Walter Sinnot-Armstrong, 'Some Varieties of Particularism', supra nota 5 .

${ }^{13}$ Desarrollo esta idea en José Juan Moreso, 'Legal Positivism and Legal Adjudication' en IVR International Seminar on Legal Theory: 'The Future of Legal Positivism'. Alicante, mayo de 2004.
} 
entonces el deber de acudir al hospital revoca el deber de cenar con mi amigo, se trata de un overrider. Como dice Walter Sinnot-Armstrong, al que se deben estas ideas y esta terminología: 'Overriders, underminers, reversers, and excuses are, then, all reason defaters'. ${ }^{14}$

Entonces, los principios morales deben ser formulados incluyendo sus defaters. Ahora bien, los defeaters siempre contendrán conceptos morales. Sólo en este sentido es posible codificar los principios morales. Es claro que, al menos, por razones epistémicas no podemos codificar todos los defeaters acudiendo únicamente a propiedades naturales. Además, dadas las infinitas descripciones posibles de las acciones, es plausible pensar que también dicha tarea es irrealizable por razones conceptuales. ${ }^{15}$ Este es el sentido en el cual el universalismo puede ser adecuado: hay principios morales, ahora bien -y esta es la concesión al particularismo- que contienen necesariamente conceptos evaluativos. ${ }^{16}$ Podemos de este modo revisar los antecedentes de nuestros principios prima facie haciéndolos más específicos, pero con el coste de incorporar en ellos, como defeaters, conceptos evaluativos.

Así es como, por otra parte, están codificadas las normas jurídicas. Si alguien mata a otro, comete un homicidio castigado, en principio, por el código penal; pero dicha acción no es punible si ha sido realizada en legítima defensa o en estado de necesidad o cualquiera del resto de las causas de justificación, o ha sido realizada en presencia de alguna de las causas de exculpación

\footnotetext{
${ }^{14}$ Walter Sinnot-Armstrong, 'Some Varieties of Particularism', supra nota 5, p. 5.

${ }^{15}$ Dicha sugerencia en Bruno Celano, 'Particolarismo, caratterizzacioni di desiderabilità, pluralismo etico. Considerazioni sulla forma del ragionamento pratico', manuscrito (Seminario de Filosofía del Derecho. Universitat Pompeu Fabra, Barcelona, marzo 2004) y José Juan Moreso, 'Dos concepciones de la aplicación de las normas de derechos fundamentales', manuscrito 2003.

${ }^{16}$ Esta es la posición de David McNaughton, Piers Rawling, 'Unprincipled Ethics' en Brad Hooker, Margaret Little (eds.), Moral Particularism (Oxford: Oxford University Press, 2000), 256-275.
} 
(trastorno mental, por ejemplo). Las causas de justificación y las excusas son los defeaters en la codificación de las normas penales. Dichos defeaters están de hecho formulados de tal modo que incorporan conceptos evaluativos (en el caso de la legítima defensa, que la agresión a la que se responde sea ilegítima, que no mediare provocación suficiente, etc. ${ }^{17}$. Si alguien realiza un contrato de compraventa, obligándose a la entrega de un coche a cambio de un precio, tal contrato es válido siempre que no medien, por ejemplo, algunos vicios del consentimiento. Es decir las obligaciones en el derecho privado están también sujetas a defaters: los contratos son nulos si son contrarios a las leyes, a la moral o al orden público, son también nulos si se otorgan con algún vicio del consentimiento (error, coacción, etc.). Como puede apreciarse, también en este caso los defeaters contienen conceptos evaluativos.

Por lo tanto, también desde un punto de vista descriptivo-explicativo (véanse las reflexiones en este sentido de la autora en p. 60), el derecho puede contemplarse como una codificación de una especie cualificada de universalismo.

No veo razones para rechazar este punto de vista para la moral, ni desde el punto de vista conceptual, ni desde el punto de vista justificativo. Ni siquiera Jonathan Dancy rechaza esta posibilidad. ${ }^{18}$ Ahora bien, Dancy replica diciendo que de este modo no conseguimos todavía dar forma (shape) a las propiedades naturales sobre las que las propiedades morales supervienen, puesto que ahora queda por averiguar sobre qué propiedades naturales supervienen los defaters. Es decir, esta forma de universalismo no es más

\footnotetext{
${ }^{17} \mathrm{He}$ desarrollado esta idea previamente en José Juan Moreso, 'Principio de legalidad y causas de justificación (sobre el alcance de la taxatividad)', Doxa 24 (2001): 532-535.

${ }^{18}$ Jonathan Dancy, 'Defending Particularism', Metaphilosophy 30 (1999): 24-32 y Jonathan, Dancy, 'Moral Particularism', Edward N. Zalta (ed.), The Stanford Encyclopedia of Philosophy (Summer 2001 edition), http://plato.stanford.edu/archives/sum2001/entries/moralparticularism/.
} 
que un particularismo disfrazado de universalismo, un lobo con piel de cordero. Es un argumento similar al de Bruno Celano. ${ }^{19}$ Celano sostiene que no hay ningún modo estable de llevar a cabo la revisión desde nuestros condicionales derrotables hasta los condicionales inderrotables y no lo hay porque la noción de una tesis de relevancia última está mal formada. La tesis de relevancia última es aquella que establece cuáles son las propiedades naturales relevantes de un modo exhaustivo ${ }^{20} \mathrm{y}$, según Celano, no hay esperanzas de alcanzar tal objetivo (vd. también las reflexiones de Redondo en pp. 43, nota 19). En palabras de Celano: 'el conjunto de las combinaciones de las propiedades potencialmente relevantes, que toman en cuenta la historia del caso, no es agotable'. ${ }^{21}$

Estas consideraciones de Celano (y de Dancy) nos llevan a otra limitación del universalismo. Concedo, sin más argumentos, que en la codificación de las normas morales (y jurídicas) no puede irse más allá de principios o normas con defaters evaluativos. Sin embargo, el discurso de aplicación de dichas codificaciones puede ir más allá. ${ }^{22}$ Sería irrazonable suponer que, enfrentados a un problema moral, debamos reconstruir todos nuestros principios morales válidos para todas las acciones humanas posibles. Es más sensato considerar que podemos adoptar un determinado universo del discurso, es decir, un conjunto determinado de acciones humanas. Por

${ }^{19}$ Bruno Celano, 'Particolarismo, caratterizzacioni di desiderabilità, pluralismo etico. Considerazioni sulla forma del ragionamento pratico', supra nota 15.

${ }^{20}$ La noción de tesis de relevancia se halla en Carlos E. Alchourrón, Eugenio Bulygin, Normative Systems (New York-Wien: Springer, 1971), cap. VI. Vd. también Jorge L. Rodríguez, Germán Sucar, 'Las trampas de la derrotablidad. Niveles de análisis de la indeterminación del Derecho' Analisi e Diritto (1998), 277-305, en p. 297; Jorge L. Rodríguez, 'Axiological Gaps and Normative Relevance', Archiv für Recths-und Sozialphilosophie 86 (2000), 151-167.

${ }^{21}$ Bruno Celado, 'Particolarismo, caratterizzacioni di desiderabilità, pluralismo etico. Considerazioni sulla forma del ragionamento pratico', supra nota 15, p. 17.

${ }^{22}$ Reformulo aquí algunas ideas ya presentadas en José Juan Moreso, 'A propósito di revisioni stabili, casi paradigmatici e ideali regolativi: replica a Celano', Ragion Pratica, 18 (2002): 241-248, en donde replicaba a Bruno Celano, 'Defeasibility' e bilanciamento. Sulla possibilità di revisioni stabili', Ragion Pratica, 18 (2002): 223-239. 
ejemplo, el conjunto de las acciones de devolver los libros que tenemos en préstamo. ${ }^{23}$ Tal vez en este caso, podamos alcanzar algo como una tesis de relevancia. Son relevantes, con seguridad, el plazo de tiempo por el que tenemos el préstamo, el hecho de que el que nos lo prestó sea su verdadero propietario (no es un libro sustraído de la biblioteca universitaria por ejemplo), debemos incluir también las justificaciones (tuvimos que ausentarnos de la ciudad por un tiempo) y las excusas (lo dejamos olvidado en un cambio de domicilio). Podemos también delimitar previamente el conjunto de normas morales a tomar en cuenta, de modo de ignorar casos recalcitrantes siempre imaginables. Podemos atender únicamente a la norma moral que obliga a devolver lo prestado bajo ciertas condiciones e ignorar, ahora, la norma moral que prohíbe inducir a otro al suicidio, y así ignorar el supuesto de que la lectura del libro, dado el estado anímico de su propietario, puede llevarlo a suicidarse. Obviamente que si apreciamos que este es el caso, debemos introducir esta norma en el sistema. Pero limitada a un universo de acciones y a un subconjunto de las normas morales, creo que es razonable aspirar a obtener una tesis de relevancia manejable. Por otro lado, así operan los juristas cuando se enfrentan a un caso real o hipotético: toman en cuenta un universo del discurso delimitado y un subconjunto de normas jurídicas (no todas las normas jurídicas, ya que no es sensato, aunque son un conjunto finito, pensar en una reconstrucción global de todas ellas). Para microsistemas así concebidos la operación de revisión estable es alcanzable y permite pasar, en este contexto delimitado, de la formulación de defeaters evaluativos a defeaters descritos mediante propiedades naturales. Obviamente que se trata, por definición, de reconstrucciones incompletas. También es verdad, como quieren los particularistas, que dichas reconstrucciones dependen del contexto, pero no dependen -como arguyen los particularistas- del contexto establecido por las circunstancias del caso concreto, sino que dependen del contexto del problema normativo

\footnotetext{
${ }^{23}$ Por tomar un ejemplo de Jonathan Dancy, Moral Reasons, supra nota 10, p. 60.
} 
seleccionado. Es decir, en un contexto normativo así delimitado, hay propiedades naturales uniforme e invariablemente relevantes.

Alguien puede considerar, con razón, que esta defensa del universalismo concede demasiado al particularismo, dado que sólo tenemos principios morales si los concebimos como incorporando conceptos evaluativos y en el razonamiento práctico el juego de estos principios está delimitado por el contexto del universo del discurso seleccionado y de los principios morales que consideramos aplicables. Sin embargo, aún con estos límites, la imagen del razonamiento práctico que obtenemos permite la subsunción de casos individuales en pautas generales y deja un razonable espacio para la articulación y la consistencia de las decisiones. Esta concepción del razonamiento práctico vale, en mi opinión, tanto para la moral cuanto para el derecho.

(V)

Estoy ahora en condiciones de abordar el dilema al que me refería en la introducción - en (I)-. Así lo plantea la autora (p. 57):

Posiciones como las de Dworkin o del Positivismo Inclusivo (que a primera vista abrazan una posición universalista) tienen que aceptar una de las siguientes tesis incompatibles entre sí. Por una parte, si ellas insisten seriamente en que las normas jurídicas pueden ser derrotables en virtud de la aplicación de normas o principios morales, ellas deberían reconocer que las así llamadas 'normas jurídicas' no constituyen razones universales. Las así llamadas 'normas jurídicas' serían sólo formulaciones provisorias de genuinas normas universales -morales- que, si aplicables a un caso objeto de decisión, pueden derrotar a las formulaciones jurídicas, impidiendo que éstas constituyan razones, o alterando dichas razones en el caso en cuestión. Esta posición es universalista, pero en relación a las 
normas morales, no a las normas jurídicas. En alternativa, si no se está dispuesto a aceptar esta consecuencia, y se insiste en que las normas jurídicas sí constituyen razones universales, entonces se tiene que admitir que los principios morales no derrotan a las normas jurídicas. Los principios morales entran en conflicto con las normas jurídicas dejando intacta su identidad y relevancia. En pocas palabras, posiciones como las de Dworkin o del Positivismo inclusivo, o bien defienden el carácter universal de las razones jurídicas, en cuyo caso las normas jurídicas no pueden ser normas derrotables, o bien defienden que las normas jurídicas son derrotables, en cuyo caso deben admitir que las únicas razones universales son las constituidas por las normas morales.

Creo que éste no es el modo en que el Positivismo inclusivo y Dworkin plantean la cuestión de las relaciones entre Derecho y moral. Y lo creo, al menos, por dos razones.

En primer lugar, la tesis de la primacía de la moral sobre el derecho en relación con su relevancia práctica es una tesis acerca del razonamiento práctico en general y su fortuna depende de las razones a favor y en contra de la unidad del razonamiento práctico. ${ }^{24}$ Aceptemos por hipótesis la tesis de la unidad del razonamiento práctico (incidentalmente, mi opinión es que se trata de una tesis plausible, pero no es éste el lugar para discutirla), ¿qué consecuencias tiene tal tesis para la justificación de las decisiones jurídicas? En mi opinión, cuando hablamos de justificación de las decisiones jurídicas podemos estar refiriéndonos, al menos, a tres cosas distintas entre sí.

\footnotetext{
${ }^{24}$ Dudas sobre la tesis de la unidad del razonamiento práctico pueden verse en $\mathrm{M}$. Cristina Redondo, La noción de razón para la acción en el análisis jurídico (Madrid: Centro de Estduios Constitucionales, 1996), cap. VI y una defensa de la tesis de la unidad en Juan Carlos Bayón, La normatividad del derecho: deber jurídico y razones para la acción (Madrid: Centro de Estduios Constitucionales, 1991), cap. 7.
} 
En primer lugar, podemos referirnos a la justificación lógica. $\mathrm{Si}$ aceptamos que existen relaciones de consecuencia lógica entre normas, entonces un razonamiento como el siguiente está lógicamente justificado:

1) El que no se levantare, al toque de corneta, a las 7 de la mañana, debe ser castigado a la pena de muerte

2) Ticio se quedó dormido el día d y no se levantó hasta las 10 de la mañana

Ticio debe ser castigado a la pena de muerte

Si la norma contenida en 1), es una norma jurídica aplicable según el sistema S, y si el contenido proposicional de 2) es verdadero, entonces la conclusión está jurídicamente justificada con arreglo al sistema jurídico $\mathrm{S}$. Este es el segundo sentido de justificación, la justificación jurídica.

Dado que esta decisión tiene relevancia moral (afecta de manera central al bienestar de Ticio), podemos también interrogarnos si está decisión está justificada moralmente. Es más, dado que por hipótesis hemos aceptado la tesis de la unidad del razonamiento práctico, es inevitable que el aplicador de 1) -si es racional- tome en cuenta si su decisión está justificada en este último sentido. Supongamos que, como yo, piensa que se trata de una norma aberrante desde el punto de vista moral, entonces la decisión de castigar a Ticio a la pena de muerte no está justificada moralmente. Es más, si el aplicador es un agente moral racional, ceteris paribus, no debe tomar esa decisión. Esto es todo lo que la tesis de la primacía de la moral sobre el derecho requiere. No veo porqué ha de ser esta una tesis controvertida, ni patrimonio exclusivo de Dworkin o del positivismo jurídico inclusivo. Creo que es una tesis de filosofía moral, bien fundada si la unidad del razonamiento práctico se mantiene. Esto es, dicha tesis, aceptada la tesis de la unidad, deviene una tesis bastante inocua: dado que los jueces son agentes morales, cuando toman decisiones que afectan al bienestar de terceros (lo que ocurre virtualmente siempre en las decisiones judiciales) deben (un deber moral) fundarlas en normas morales. ${ }^{25}$

${ }^{25}$ Había desarrollado este argumento en José Juan Moreso, 'Comanducci sobre (neo)constitucionalismo’, Isonomía 19 (octubre 2003): 267-282, en pp. 279. 
En este sentido, cuando lo que requiere el derecho es contrario a lo requerido por la moral, la identidad de las normas jurídicas queda intacta, pero su relevancia práctica queda afectada. ${ }^{26}$ Una cosa distinta, que es la que creo preocupa a los positivistas inclusivos y a Dworkin, es que la legislación de un sistema jurídico castigue con la pena de muerte determinados delitos, pero la Constitución prohíba los castigos crueles (como es el caso en los Estados Unidos) o los castigos inhumanos y degradantes. Entonces, para saber lo que el derecho requiere deberá establecerse si la pena de muerte es o no cruel y ello, en mi opinión, no puede establecerse más que acudiendo a la argumentación moral.

Y esto nos lleva al segundo punto al que quiero referirme. La propiedad, establecida mediante el concepto evaluativo cruel funciona como un defeater, de carácter jurídico, que hace de los castigos previstos, castigos excluidos constitucionalmente. ¿Cómo pasar de este defeater jurídico -pues está previsto en la Constitución- pero cuyo contenido sólo puede ser identificado mediante la argumentación moral? Es ahora relevante lo que decía en (IV)

\footnotetext{
${ }^{26}$ Sólo, tal vez, los iusnaturalistas desafiarían esta conclusión, si es que hacen depender la validez jurídica de una norma de su conformidad a la moral. Sobre si este es o no el núcleo del iusnaturalismo puede verse, recientemente, Bruno Celano, 'Giusnaturalismo, positivismo giuridico e pluralismo etico' en Positivismo giuridico e diritto naturale agli inizi del XXI secolo, Dipartimento di Cultura Giuridica 'Giovanni Tarello' (Genova, maggio 2004) y Timothy Endicott, 'Natural Law Theory' en Positivismo giuridico e diritto naturale agli inizi del XXI secolo, Dipartimento di Cultura Giuridica 'Giovanni Tarello' (Genova, maggio 2004). Tampoco Ronald Dworkin piensa que el derecho esté siempre de acuerdo con la moralidad (Ronald Dworkin, 'Introduction: The Moral Reading and the Majoritarian Premise', en Ronald Dworkin, Freedom's Law (Cambridge, Mass.: Harvard University Press, 1996, 138), p. 36: 'Yo no leo la Constitución como si contuviera todos los principios importantes del liberalismo político. En otros escritos, por ejemplo, he defendido una teoría de la justicia económica que requeriría una redistribución substancial de la riqueza en las sociedades políticas opulentas. Algunas constituciones nacionales intentan establecer un grado de igualdad económica como un derecho constitucional, y algunos juristas americanos han arguído que nuestra Constitución puede ser comprendida como estableciéndolo. Pero yo no pienso de este modo, por el contrario, he insistido en que la integridad detendría cualquier intento de argumentar desde las cláusulas morales abstractas de la declaración de derechos, o desde cualquier otra parte de la constitución, hasta tal resultado' (notas al pie omitidas).
} 
acerca de la delimitación del universo del discurso: son propiedades naturales diversas las que hacen crueles castigos tan diversos entre sí como: los castigos corporales, las penas de cárcel o las multas, lo que hace cruel un castigo a un menor puede no hacerlo a un adulto, etc. Así podemos pensar, por ejemplo, en cuáles son las propiedades que hacen crueles las penas de privación de libertad a los menores de edad. Pues bien, esta es la relevancia de la moralidad en el derecho: para pasar de los principios y normas jurídicas, que contienen conceptos evaluativos, a premisas que puedan ser adecuadas en el razonamiento jurídico justificativo, es necesario -a menudo- acudir al razonamiento moral. No se trata en este caso de que las normas jurídicas sean derrotadas por normas morales, sino que el contenido de las normas jurídicas ha de ser identificado mediante el uso del razonamiento moral.

Por lo tanto, en contra de lo sugerido por Redondo, las normas jurídicas -cuando están de acuerdo con la moralidad- disfrutan del universalismo moderado que he defendido, un universalismo sometido a dos límites importantes: por una parte están formuladas de modo que contienen defeaters con contenido evaluativo y, por otra parte, sólo en contextos de aplicación adecuadamente diseñados pueden establecerse el conjunto de propiedades naturales que tienen, en dicho contexto, una relevancia uniforme e invariable. O dicho de otro modo, en mi opinión la autora confunde dos problemáticas distintas acerca de las relaciones entre el derecho y la moral: una cosa es qué ocurre cuando lo que el derecho requiere está en contra de la moral y otra qué ocurre cuando el derecho remite a la moralidad. Las tesis del positivismo inclusivo y de Dworkin se refieren al segundo problema y son, en mi opinión, compatibles con la vindicación de una concepción universalista moderada tanto del razonamiento jurídico como del razonamiento moral. 